\title{
Search behavior in cats and dogs: Interspecific differences in working memory and spatial cognition
}

\author{
FRANÇOIS Y. DORÉ, SYLVAIN FISET, SONIA GOULET, \\ MARIE-CHANTALE DUMAS, and SYLVAIN GAGNON \\ Université Laval, Quebec, Quebec, Canada
}

\begin{abstract}
Cats' and dogs' search behavior was compared in different problems where an object was visibly moved behind a screen that was then visibly moved to a new position. In Experiments 1 (cats) and 2 (dogs), one group was tested with identical screens and the other group was tested with dissimilar screens. Results showed that in both species, search behavior was based on processing of spatial information rather than on recognition of the visual features of the target screen. Cats and dogs were unable to find the object by inferring its invisible movement. They reached a high level of success only if there was direct perceptual evidence that the object could not be at its initial position. When the position change was indicated by an indirect cue, cats searched more at the object's initial than final position, whereas dogs searched equally at both positions. Interspecific similarities and differences are interpreted in terms of the requirements for resetting working memory.
\end{abstract}

It has repeatedly been shown in Piagetian visible displacement tests of object permanence that cats (Doré, 1986; Goulet, Doré, \& Rousseau, 1994; Thinus-Blanc, Poucet, \& Chapuis, 1982; Triana \& Pasnak, 1981) and dogs (Gagnon \& Doré, 1992, 1993, 1994; Triana \& Pasnak, 1981) are highly successful in problems where they have to find an object they saw move and disappear behind a screen or a succession of identical screens. On the other hand, the performance of both species is quite different in invisible displacement tests where the position change of the object is invisible. In these tests, the target object is first hidden in a container that is immediately moved behind a screen. While the container is still behind the screen, the object is removed from it and concealed behind the screen. Finally, the empty container is removed from the target screen and shown to the subject, thus providing an indirect visual cue that the object's position was changed from the container to the back of the screen. Dogs are able to solve this kind of problem, but their level of success is lower than it is in visible displacement problems (Gagnon \& Doré, 1992, 1993). Cats are unable to solve invisible displacement tests

This research was supported by Grant OGP0007030 from the Natural Sciences and Engineering Research Council (NSERC) of Canada to F.Y.D. It received approval from the Comité de protection des animaux de laboratoire de l'Université Laval, which is responsible for the application and enforcement of the rules of the Canadian Council on Animal Care. The authors thank M. Thibault, C. Bouchard, and M. Wampach for their assistance in collecting data as well as the dogs' owners and breeders who participated in Experiment 2. They also thank two anonymous reviewers for their constructive comments. Correspondence should be addressed to F. Y. Doré, Laboratory of Neuropsychology, NIMH, 9000 Rockville Pike, Building 49, Room 1B80, Bethesda, MD 20892.

-Accepted by previous editor, Vincent M. LoLordo
(Doré, 1986, 1990; Dumas \& Doré, 1989; Goulet et al., 1994; Pasnak, Kurkjian, \& Triana, 1988).

Recently, Goulet et al. (1994) examined cats' search behavior in standard Piagetian tests of object permanence (Experiments 1 and 2) as well as in a new situation of invisible displacement (Experiments 3 and 4) where there was no container and the problem was, in principle, easier to solve. In the new procedure, the object first disappeared behind a screen; a few seconds later, the object reappeared behind a different screen and then disappeared at this new location. Indications that the object was moved from the first to the second screen were given. The experimenter simulated the movements that she would normally make to produce the position change of the object, these movements serving as a cue as to where to expect the object's reappearance. She also either simulated the noise that cats had earlier learned to associate with the displacement of the object or she showed, just before the simulated displacement, that the first hiding screen was empty. The percentages of search attempts made at the object's initial and final locations did not differ. These results were consistent with the predictions made from cats' performance in Piagetian invisible displacement tests and confirmed that these animals are able to represent the position changes only of an object they have directly perceived.

According to Goulet et al.'s (1994) interpretation of cats' search behavior, when an object is visibly hidden at two successive locations, working memory is not totally reset between the first and the second disappearance. The information on the prior hiding location is activated to some degree, but the final hiding location from which the object was not visibly removed is the most activated information. In other words, the disappearance of an object behind a screen activates this location in working memory, and the 
activation remains strong as long as the object is not visibly removed from it. In invisible displacements, the object is not visibly removed from either the first or the second hiding location. Consequently, in spite of perceptual cues indicating that the object was transferred from one position to the other, the initial location is more activated in memory than the final location (Piagetian invisible displacement tests) or both locations remain strongly activated (new procedure).

The empirical investigation of working memory and spatial cognition underlying search behavior is less advanced in dogs than it is in cats. However, it seems that in dogs, resetting of working memory is based not only on direct evidence (e.g., removal of the object from the hiding screen in Piagetian visible displacement tests) but also on indirect perceptual cues. As already mentioned, dogs succeed to some degree in Piagetian invisible displacement tests even though the transfer of the target object from the container to the hiding screen is signaled only indirectly by showing the empty container after its movement behind the hiding screen.

The two experiments reported in the present article were aimed at analyzing in cats (Experiment 1) and in dogs (Experiment 2) the requirements for the resetting of working memory when a hidden object occupies two successive locations in the course of a single trial. A testing procedure based on Sophian's (1985) methodology for human infants was used. In this procedure, which will be referred to as the "invisible transposition problem," an object is visibly moved and hidden behind a screen, and then this screen is visibly moved to a new position. As in Piagetian invisible tests of object permanence, the object is invisibly moved from one location to another. On the other hand, the problem is simpler because the object always remains behind the screen where it disappeared, and this screen always remains in the subject's full view.

One group of subjects was tested with identical screens on three different types of invisible transposition problems in which the target screen and an empty screen were moved. In one type of transposition, the two screens were simultaneously moved to each other's positions. Hence, there was no perceptual change resulting from the manipulation. For spatial working memory to be reset, subjects had to infer the invisible movement of the hidden object from the visible movement of the target screen. In another type of problem, the initial position of the target screen was filled with the empty screen. Because the configuration formed by the hiding screens was modified by the manipulation, an indirect visual cue of the object's position change was available and could be used to reset spatial working memory. In still another type of invisible transposition, the initial position of the target screen was left empty after the manipulation. Thus, in addition to the indirect visual cue (modification of the screen configuration), there was a direct visual cue indicating that the object was no longer at its initial position.

We predicted that cats would succeed only in the last type of invisible transposition because in these animals, an object's initial hiding location is significantly deactivated in working memory only if there is direct evidence that the object's position has changed. Dogs should also succeed in problems with indirect visual cues, at least to some degree, because they are able to use the empty container in the Piagetian invisible displacement test as a cue to reset working memory. If spatial working memory in dogs can be reset not only by indirect visual cues but also by in ferrence of the invisible movement of the hidden object, they should be able to solve all three types of transposition problems.

Cats and dogs could fail the three types of invisible transpositions not because the object's initial hiding position is still strongly activated in working memory after the manipulation, but because, as Sophian (1985) suggested, their attentional capacities are too limited to cope with the movement of two screens. Therefore, we included one additional type of transposition in which only one screen was moved instead of two. If the attentional interpretation is right, performance should be better in these problems than in the preceding ones.

Another group of subjects was tested on the same types of transposition but with visually different screens instead of identical screens. Search behavior in these subjects could be based on recognition of the visual features of the hiding locations themselves rather than on encoding and retention of spatial information. If it was, cats and dogs in this group should both be successful in all types of transposition.

\section{EXPERIMENT 1}

\section{Method}

\section{Subjects}

Twelve adult cats (Felis catus, 7 males and 5 females) purchased from a laboratory supplier were used in this experiment. They stayed 21 days in the central animal care facility of the university, where they received a health examination, were treated for endo- and ectoparasites, and were vaccinated. They received a normal daily food ration and fresh water. A quarter of the food ration was eaten during experimental sessions, the rest of it being available immediately after a session.

\section{Apparatus}

The bare experimental room (Figure 1) was painted white. Part of the room's floor was covered with a gray plywood stand $(158 \mathrm{~cm}$ wide $\times 36 \mathrm{~cm}$ high $\times 215 \mathrm{~cm}$ deep) and there was an empty space at each end of the room. The experimenter who was performing the manipulations $\left(E_{1}\right)$ sat in the pit just behind the test platform. The other experimenter $\left(E_{2}\right)$, who was restraining the subject during the manipulations, sat in the other empty space.

A wall separated the stand from the subject's starting position and from the pit where $E_{2}$ was located. A holding box $(45 \mathrm{~cm}$ high $\times$ $33 \mathrm{~cm}$ deep) was fixed perpendicular to the wall. One opening of the box placed at the center of the wall was connected to the stand by a sliding door. A transparent Plexiglas door closed the opening during manipulations of an object, and an opaque white Plexiglas door was used between trials. $\mathrm{E}_{2}$ sat behind the holding box, held the cat by passing his/her arms through the other opening of the box, and watched the subject through the transparent top of this box. Above this box, a window made of transparent Plexiglas was embedded in the wall, so $E_{2}$ could see the whole apparatus. Both experimenters had access to the stand through a small concealed door on the left of the wall. 


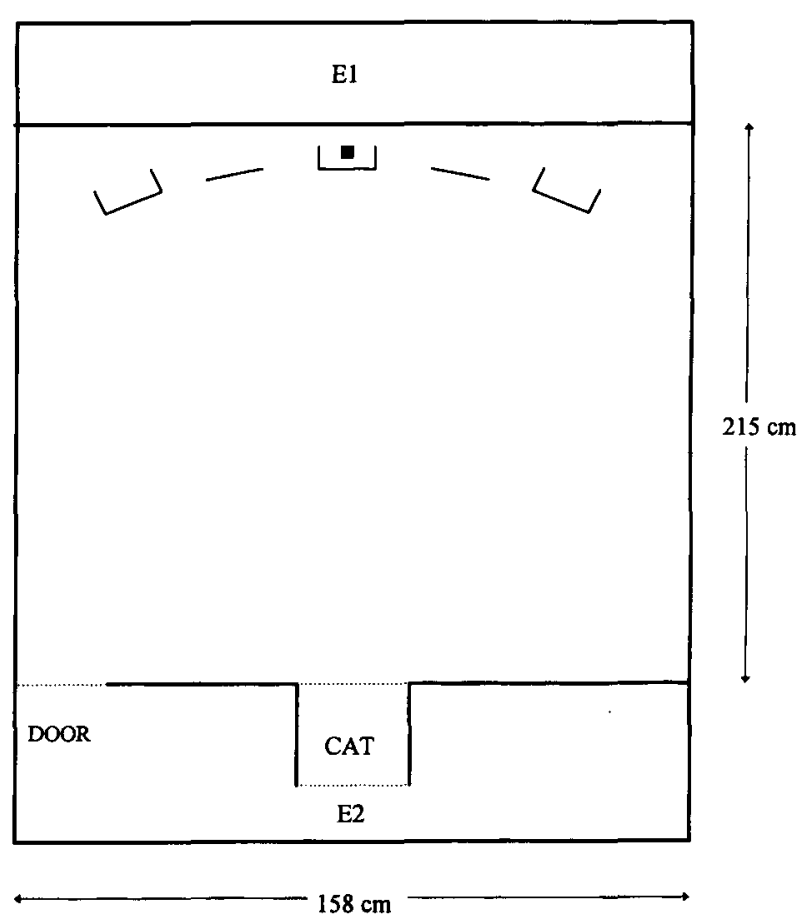

Figure 1. Experimental room and apparatus in Experiment 1 $\left(E_{1}\right.$, experimenter who performed the manipulations; $E_{2}$, experimenter who held the cat).

The target object was a red wooden cube $\left(64 \mathrm{~cm}^{3}\right)$ with a metal ring on its top and a piece of cloth on its bottom. A bent stick, terminated at one end by a hook that could be inserted into the metal ring of the cube, was used to move the target object silently. Two sets of three screens served to hide the object. All six screens were rectangular opaque white boxes with three sides and a top, but without bottom and back panels. In one set, all screens were identical ( $15 \times$ $24 \times 7.5 \mathrm{~cm})$. The screen dimensions in the other set were different $(15 \times 12.0 \times 7.5 \mathrm{~cm} ; 15 \times 24.0 \times 7.5 \mathrm{~cm} ; 15 \times 36.0 \times 7.5 \mathrm{~cm})$. The visual cues displayed on the front panels of these screens were also different. Two black stripes $(1.8 \mathrm{~cm}$ wide) were painted on the front panel; the stripes on the short, medium, and tall screens were vertical, horizontal, and diagonal, respectively. The three screens could be placed in five equally spaced positions, with one position (Position 3 ) in front of the holding box. These positions were arrayed in a semicircle at a distance of $17 \mathrm{~cm}$ from each other and were equidis$\operatorname{tant}(1.3 \mathrm{~m})$ from the subject.

\section{Procedure}

The experiment was divided into three successive steps: shaping, visible displacement training, and testing.

Shaping. The cube used as the target object did not provide olfactory cues that would be available with food, but shaping of the response was necessary. The first step, divided into several sessions of 15 min each, consisted of reinforcing cats with food when they simply approached the cube thrown in front of them. Gradually, the response criterion became more demanding until touching the object with the paw was the only reinforced response. In the second step of shaping, sessions were divided into 30 discrete trials where the animal had to touch the object near each of the three identical white screens, but never behind them. On average, subjects needed 13.1 trials $(S E=$ 6.6) to reach a criterion of 9 successes out of 10 trials.

Visible displacement training. After shaping, subjects were divided into two equal groups $(n=6)$ that were equivalent in terms of the mean number of trials required to achieve the shaping criterion. The set of identical screens was used with Group I, and the set of different screens was used with Group D. All subjects were trained to find the target object behind one of the screens. Only the three central positions (Positions 2, 3, and 4) were used in visible displacement training. In Group D, the locations of the three screens on these central positions varied from trial to trial.

At the beginning of a trial, the object was placed at a point located in front of Position 3, and $E_{2}$ restrained the animal in the holding box by grasping its front shoulders. $E_{1}$ placed the hook of the bent stick in the ring of the cube, shook the object to catch the subject's attention, moved it visibly in front of the row of screens, hid the cube behind the target screen, and withdrew the stick out of sight into the pit. Once the cube had disappeared, $E_{1}$ looked at $E_{2}$ in order to prevent cuing. Then, $E_{2}$ opened the sliding door of the holding box and the cat was released to search for the cube. If no search attempt was made in the minute that followed the subject's release (no choice), the cat was called back into the holding box for the beginning of the next trial. If the animal found the object behind the target screen and touched it with its paw before the end of the interval (success), it was called back into the holding box and was reinforced with a piece of food. However, if the subject chose another screen than the true hiding location (error), the trial was immediately interrupted, without a chance to search for the object behind a second screen (noncorrection procedure). Each session included 24 trials (three positions $X$ eight repetitions) and training was ended when the subject reached a criterion of 20 successes out of 24 trials.

Testing. A series of eight daily sessions began the day after the last visible displacement training session. The general procedure was identical to the one used in training. Groups I and D were tested with the three identical screens and the three different screens, respectively. However, unlike in training, the three screens did not always occupy the central positions and subjects were not released immediately after the object's disappearance. Once the object was hidden, subjects observed a manipulation where one or two screens were moved laterally or they had to await the end of a short delay aimed at equating the duration of the manipulation in all types of trials. Only when the manipulation or the delay was ended were the subjects released.

Four types of experimental trial and two types of control trial were used in testing. Figure 2 illustrates for each type of trial the positions of the screens at the beginning of a trial and the manipulation that was performed (before), as well as the positions of the screens at the end of the manipulation (after).

In SW (switch transposition) trials, the target screen and an empty screen were simultaneously moved to each other's positions. In SB (substitution transposition) trials, the target screen was moved to an empty position while an adjacent screen was simultaneously moved to where the target screen had been initially. As in switch transpositions, the initial position of the target screen was filled by an empty screen at the end of the manipulation. However, the configuration formed by the screens was not the same before and after the manipulation, unlike switch transpositions.

In the following two types of trials, the configuration formed by the screens was changed by the manipulation, but the initial position of the target screen was left empty at the end of the manipulation. In DO (double transposition) trials, an empty screen was moved to an adjacent position while the adjacent target screen was simultaneously moved to where the empty screen had been initially. In SI (single transposition) trials, only the target screen was moved.

In CM (control for movement) trials, a screen was moved after the object's disappearance, as in single transpositions, but the position of the target screen did not change. These trials were aimed at testing whether cats' performance was influenced by the position change of the target screen itself or by the movement of any screen. In all the preceding types of trial, screens were moved after the object's disappearance; this movement might have influenced subjects' 


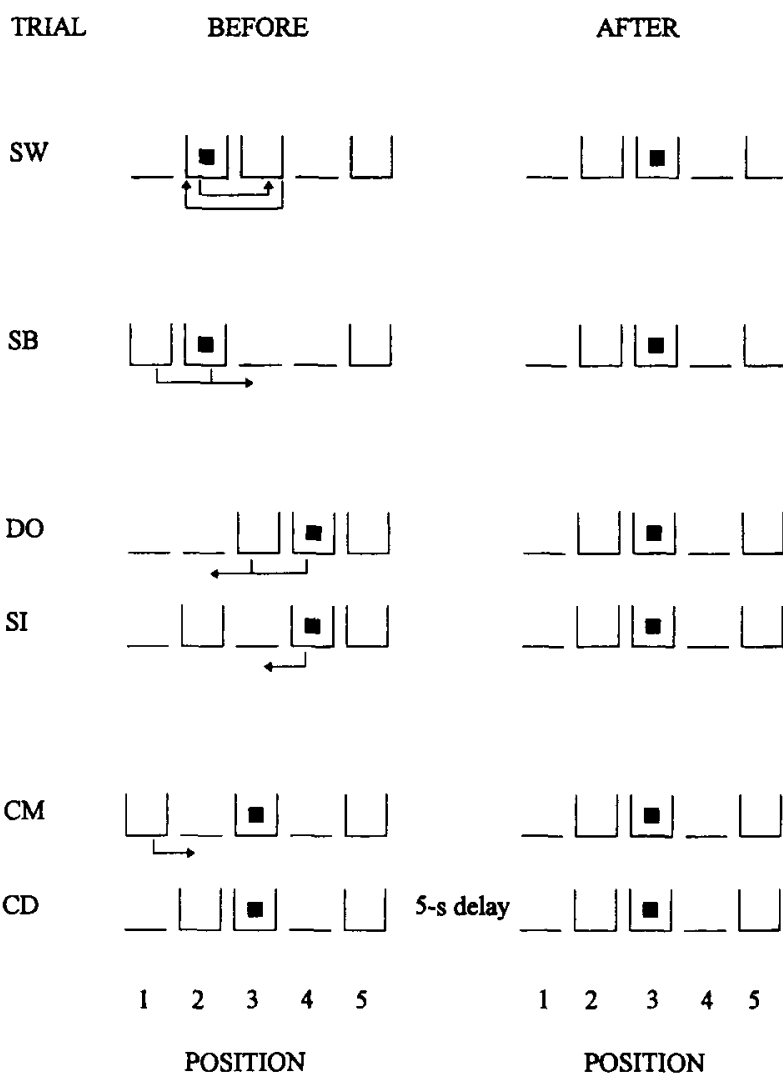

Figure 2. Schematic representation of the six types of trials before and after the manipulation. (The arrows illustrate the manipulation to be performed.) $\mathrm{CD}$, control for delay; $\mathrm{CM}$, control for movement; SI, single transposition; DO, double transposition; SB, substitution transposition; SW, switch transposition.

search behavior by creating retroactive interference with the representation in working memory of the object's location. In CD (control for delay) trials, there was no movement after the object's disappearance, and the positions of the three screens did not change within the course of a trial. However, there was a delay of $5 \mathrm{sec}$ between the object's disappearance and the release of the subject. This delay was estimated to be the maximum time required to do the manipulation in the other trials.

At the end of each of the six types of trials, the three screens were distributed on the five positions according to the same spatial configuration. There were four of these final configurations. There were two final configurations, each a mirror image of the other, in which the target screen was at the central position after the manipulation. One of them is illustrated in Figure 2. There were also two mirror image configurations in which the target screen was at one end of the array after the manipulation. In these four spatial configurations, the target screen was adjacent to an empty screen that was itself separated from the other empty screen by one position. They were the only configurations that could be produced by all six manipulations required by the four types of experimental trials and the two types of control trials.

A testing session included 24 trials that were separated by 30 -sec intertrial intervals. There were 16 trials from one of the four types of experimental trials ( 4 trials $\times 4$ final configurations) and 4 trials from each of the two types of control trials (CM and CD trials). A given type of experimental trial was tested on two consecutive daily sessions, and the order of presentation of the four kinds of sessions was randomized for each subject. Thus at the end of the eight testing sessions, each type of trials had been presented 32 times, 8 times with each of the four final configurations.

\section{Results and Discussion}

In the preliminary visible displacement training, the criterion of 20 successes out of 24 trials was reached in an average of 7.4 sessions $(S E=1.6)$ by Group I and 7.2 sessions $(S E=1.4)$ by Group D. During testing, subjects were highly motivated to search for the hidden object: A search attempt was not made in the minute following the object's disappearance (no choice) in only 1 of the 2,304 experimental and control trials.

\section{Percentage of Success}

Figure 3 shows the percentage of successes for each group in each of the six types of trial. First, it is quite clear that the performances of Group I and Group D did not differ. Second, the level of success was high in the two types of control trials (CM and CD trials) as well as in single (SI) and double (DO) transpositions, and it was much lower in switch (SW) and in substitution (SB) transpositions. An analysis of variance (ANOVA) (group $\times$ type of trials) confirmed these results. The effects of group $[F(1,10)=0.11]$ and interaction $[F(5,50)=0.13]$ were not significant, but the effect of type of trials was significant $[F(5,50)=31.95$, $p<.0001]$. A Newman-Keuls test $(p<.05)$ revealed that the levels of success in substitution and switch transpositions, which did not differ, were significantly lower than the levels of success in all the other types of trials. It also showed that the percentages of success in CD and in CM trials did not differ and that the performances in single and in double transpositions, which did not differ, were both significantly better than the one in CM trials.

The absence of a difference between Groups I and D is consistent with Fiset and Doré's $(1990,1993)$ conclusion that in this kind of situation, cats locate and find the hidden object by encoding and maintaining in working memory spatial information rather than by recognizing visual features of the target screen. The results also show that an interpretation in terms of limited attentional capacities is unsatisfying and that cats were able to cope with the

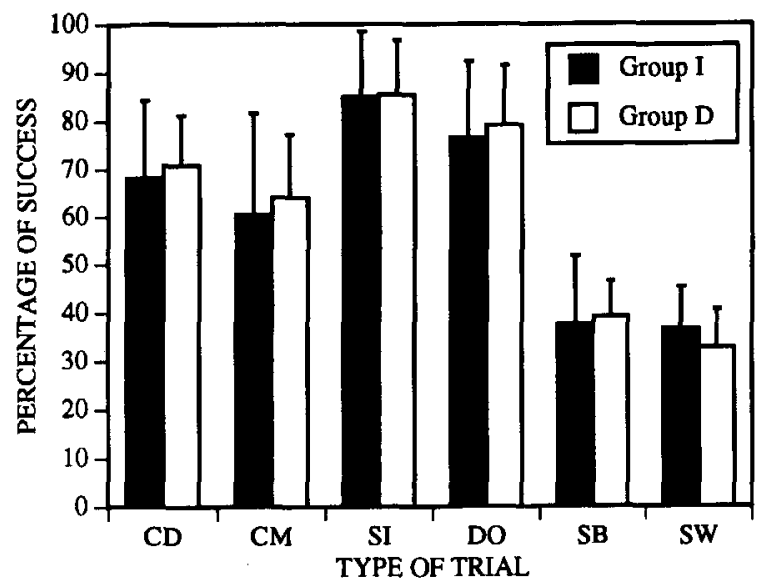

Figure 3. Mean percentage of success of Group I (identical screens) and Group D (different screens) in the six types of trials of Experiment 1 with cats. CD, control for delay; CM, control for movement; SI, single transposition; DO, double transposition; SB, substitution transposition; SW, switch transposition. 
movement of two screens. Although performance in single transpositions was significantly better than in substitution and in switch transpositions, it did not differ from performance in double transpositions, which involved the simultaneous movement of the same two screens. Also unsatisfying is an interpretation based on retroactive interference created by the movement of the screens after the object's disappearance, because the percentage of success in CM trials did not differ from that in CD trials. The poorer performance in CM trials compared with single or double transpositions may be related to the distribution of the different types of trial within a session. Screens were moved in 20 trials out of 24 , and in 16 of these 20 trials, the moved screen(s) included the target screen. It is possible that in CM trials, subjects associated the movement of an empty screen with the target screen.

Cats' lower performance in substitution and in switch transpositions can be explained by the nature of the perceptual cues available for resetting working memory. In single and in double transpositions, the initial position of the target screen was left empty after the manipulation, and there was thus direct perceptual evidence that the hidden object could no longer be at its initial position. This information did not in itself reveal where to search for the object, but it deactivated the representation of its initial position in working memory. In substitution and in switch transpositions, the initial position of the target screen was filled with the adjacent screen and there was no direct perceptual cue after the manipulation. If the modified configuration of screens in substitution transpositions served as an indirect perceptual cue for resetting working memory, cats did not use it.

\section{Error Distribution}

Because the percentages of success in Groups I and D did not differ, and the error distributions in these two groups

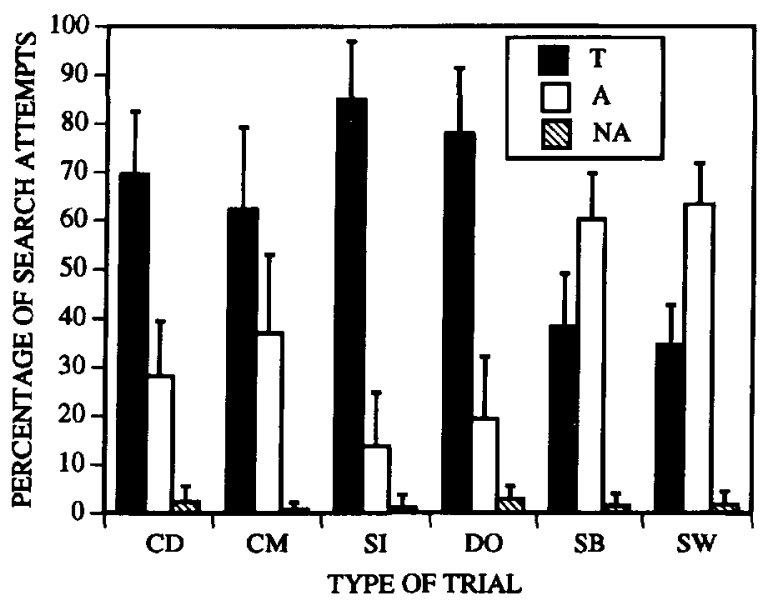

Figure 4. Mean percentage of search attempts at the target screen ( $T$ ), at the adjacent screen (A), and at the nonadjacent screen (NA) in the six types of trials of Experiment 1 with cats. CD, control for delay; CM, control for movement; SI, single transposition; DO, double transposition; SB, substitution transposition; SW, switch transposition. were very similar, their data were pooled in the following analysis. Figure 4 presents for each type of trial the percentages of search attempts made at each of the three screens. (T refers to the target screen; A refers to the empty screen that was adjacent to the target screen after the manipulation; and NA refers to the empty screen that was not adjacent to either the target screen or the other screen.) Far more unsuccessful search attempts were made at $A$ than at NA. In CM trials, double (DO), substitution (SB), and switch (SW) transpositions, A was moved during the manipulation, but in CD trials and in single (SI) transpositions, it was not moved. Therefore, cats' errors were not influenced by movement of this screen but by proximity to the target screen.

The distribution of search attempts at $T$ was compared with that at $\mathrm{A}$ in double, substitution, and switch transpositions, all of which involved two simultaneous movements, by an ANOVA (type of trial $\times$ choice) in a randomized block design (the error sum of squares was partitioned). This analysis showed that the interaction was significant $[F(2,22)=47.52, p<.0001]$. An analysis of simple main effects (with adjusted degrees of freedom for within-subject designs, as recommended by Howell, 1988) revealed that in double transpositions, significantly more search attempts were made at $\mathrm{T}$ than at $\mathrm{A}[F(1,30)=85.94, p<.0001]$, whereas in substitution transpositions and in switch transpositions, more search attempts were made at $\mathrm{A}$ than at $\mathrm{T}$ $[F(1,30)=12.0, p<.002$, and $F(1,30)=20.60, p<$ .0001 , respectively].

Thus, when there was direct perceptual evidence that the object could not be at its initial position (double transpositions), working memory was reset and cats generally searched for the object at its true location. It seems that they chose this position not only because it was the nearest to the object's initial position but also because the movement was made in this direction. Otherwise, in the double transpositions (DO) illustrated in Figure 2, subjects would have made as many search attempts at $\mathrm{T}$ as at $\mathrm{NA}$, and they did not $(\mathrm{T}=81.9 \% ; \mathrm{NA}=4.7 \%)$.

When the perceptual cue indicating that the object's position had changed was only indirect (substitution transpositions), or when there was no cue that such change had occurred (switch transpositions), cats tended to search for the hidden object at A rather than at T. Therefore, the object's initial position was more activated in working memory than its final position.

\section{EXPERIMENT 2}

In Experiment 2, dogs were tested on the same types of invisible transposition as the ones in Experiment 1.

\section{Method}

\section{Subjects}

Twenty purebred adult dogs (Canis familiaris, 15 females and 5 males) that belonged to private owners and breeders, were used in this experiment. All of them came from breeds classified as hunting dogs by the American Kennel Club. There were 1 English cocker, 5 American cockers, 2 golden retrievers, 4 Gordon setters, 6 Labradors, 1 longhaired dachshund, and 1 hard-haired dachshund (mean age, 3 years, 
5 months; range, 1.5-8 years). Gagnon and Dore (1992) have shown that there is no interbreed difference of performance in this kind of task.

\section{Apparatus}

The experiment was conducted in the owner's or breeder's house, where a room was selected on the basis of three criteria: an area of at least $4 \mathrm{~m}^{2}$, the quality of illumination, and the dog's familiarity with the environment (daily contact).

In order to maintain subjects' attention and motivation, several different squeezable rubber toys (height, $9 \mathrm{~cm}$ ) of various shapes and colors were used as the target object during the experiment. Each toy was manipulated by a $1.25-\mathrm{m}$ transparent nylon thread tied to it. The screens used to hide the object were three rectangular opaque white boxes with three sides and without back panels. The bottoms of the screens were filled with lead bars to make them more stable, and an edge on the back of the screen prevented the object from slipping out when the screens were moved. There were two sets of three screens each. In one set, the screens were identical $(17 \mathrm{~cm}$ wide $\times 30 \mathrm{~cm}$ high $\times 12 \mathrm{~cm}$ deep). In the other set, the screen dimensions differed $(17 \times 15 \times 12 \mathrm{~cm} ; 17 \times 30 \times 12 \mathrm{~cm} ; 17 \times 45 \times 12 \mathrm{~cm})$. The orientation of the two black stripes $(2 \mathrm{~cm}$ wide) displayed on the front panels also differed: Vertical, horizontal, and diagonal stripes were displayed on the small, medium, and tall screens, respectively. The screens were fixed on a transparent plastic carpet with Velcro bands and they could be placed on five equally spaced locations; Position 3 was in front of the dog's starting position. These positions were arrayed in a semicircle at a distance of $28 \mathrm{~cm}$ from each other and were equidistant $(2 \mathrm{~m})$ from the subject.

Even though Gagnon and Doré (1992) have shown that olfaction does not help in solving this kind of problem, olfactory cues were masked with a solution of rose water diluted in tap water $(1: 10)$ that was sprayed on the objects, the screens, and the carpet. This substance was chosen because it is highly odoriferous, edible, and nontoxic. Two fans placed at $40 \mathrm{~cm}$ of each extremity of the plastic carpet spread the rose water odor uniformly.

\section{Procedure}

Shaping. During shaping, subjects had to display one of the following responses: grasping the object with the mouth, touching it with the paw, or touching it with the muzzle. After each successful trial, the subjects were reinforced with bits of dry commercial food or pieces of dog cookies. Every subject reached the criterion of 9 successes out of 10 trials in the first 10 trials.

Visible displacement training. After shaping, subjects were divided into two equal groups $(n=10)$. The set of identical screens was presented to Group I and the set of different screens was presented to Group D. As in Experiment 1, only the three central positions $(2,3$, and 4$)$ were used in visible displacement training, and in Group D, the locations of the three screens on these central positions varied from trial to trial. The procedure was similar to that used in Experiment 1, although adjustments had to be made because the experiment took place in the owners' or breeders' houses.

At the beginning of a trial, $E_{1}$, who stood behind the central screen, placed the object in front of Position 3 by manipulating it with the nylon thread. $E_{2}$ manually restrained the dog by holding its front shoulders, thus preventing the initiation of any movement toward the object or a particular screen. $E_{1}$ caught the subject's attention by shaking the object with the nylon thread, moved the ubject visibly in front of the row of screens, and hid it behind one of the screens. When the object was totally hidden and $E_{1}$ was motionless, $E_{1}$ looked at $E_{2}$ to prevent cuing and $E_{2}$ released the subject. A trial was failed if the animal chose the wrong screen or made no search attempt in the minute that followed disappearance (noncorrection procedure). If the animal found the object behind the target screen, it was called back by $E_{2}$ and was reinforced with a piece of food. Each session included 24 trials ( 3 positions $\times 8$ repetitions) and training was ended when the subject reached a criterion of 20 successes out of 24 trials.
Testing. In Experiment 2, the testing procedure was the same as that in Experiment 1, except for the modifications described for visible displacement training.

\section{Results and Discussion}

In the preliminary visible displacement training, all dogs reached the criterion of 20 successes out of 24 trials in the first session, that is, faster than the cats in Experiment 1. During testing, dogs were also highly motivated to search for the hidden object: A search attempt was not made in the minute following the object's disappearance (no choice) in only 7 of the 3,840 experimental and control trials.

\section{Percentage of Success}

Figure 5 shows the percentage of success for each group in each of the six types of trial. First, as in Experiment 1, it is quite clear that the performances of Groups I and D did not differ. Second, the level of success was very high in the two types of control trials (CD and CM) as well as in single (SI) and double (DO) transpositions. It was much lower in substitution transpositions (SB) and still lower in switch transpositions (SW).

An ANOVA (group $\times$ type of trials) revealed that the effects of group $[F(1,18)=0.63]$ and the interaction $[F(5,90)=1.0]$ were not significant, but the effect of type of trials was significant $[F(5,90)=169.6, p<.0001]$. A Newman-Keuls test $(p<.01)$ confirmed that the levels of success in control trials (CD and CM trials), single (SI) transpositions, and double (DO) transpositions, which did not differ, were significantly higher than in substitution (SB) and in switch (SW) transposition trials. Performance was also significantly better in substitution transpositions than in switch transpositions.

Like cats, dogs solved this kind of problem by encoding spatial information in working memory rather than by recognizing the visual features of the target screen. For the same reasons as in Experiment 1, interpretations based

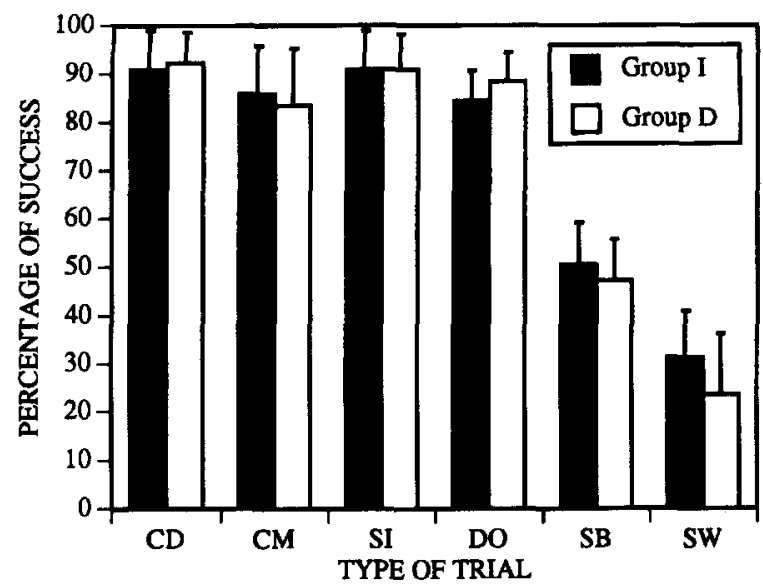

Figure 5. Mean percentage of success of Group I (identical screens) and Group $D$ (different screens) in the six types of trials of Experiment 2 with dogs. CD, control for delay; $\mathrm{CM}$, control for movement; SI, single transposition; DO, double transposition; SB, substitution transposition; $S W$, switch transposition. 
on limited attentional capacities or retroactive interference are unsatisfying, and the results are better explained by the nature of the cues available for resetting working memory.

Dogs were less successful when the initial position of the target screen was filled with the adjacent empty screen (substitution and switch transpositions) than when direct perceptual evidence indicated that the object could no longer be at the position where it had disappeared (single and double transpositions). Unlike cats, dogs performed better if the initial and final configurations of screens were different (substitution transpositions) than if they were identical (switch transpositions). It seems that dogs were able to use, at least to some degree, an indirect perceptual cue indicating that the screens' positions had changed.

\section{Error Distribution}

Figure 6 presents for each type of trial the percentages of search attempts made at the target screen $(T)$, at the empty adjacent screen (A), and at the empty nonadjacent screen (NA). As in Experiment 1, data from Groups I and $\mathrm{D}$ were pooled in the following analysis.

More unsuccessful search attempts were made at A than at NA. The distribution of search attempts at the target screen was compared with that at the adjacent empty screen in double (DO), substitution (SB), and switch (SW) transpositions by an ANOVA (type of trials $X$ choice) in a randomized block design. The interaction was significant $[F(2,38)=190.23, p<.0001]$. The analysis of simple main effects confirmed that in double transpositions, more search attempts were made at $\mathrm{T}$ than at $\mathrm{A}[F(1,56)=$ $322.40, p<.0001]$, whereas in switch transpositions, fewer search attempts were made at $\mathrm{T}$ than at $\mathrm{A}[F(1,56)=$ $110.73, p<.0001]$. On substitution transpositions, the percentages of search attempts made at the two screens did not differ.

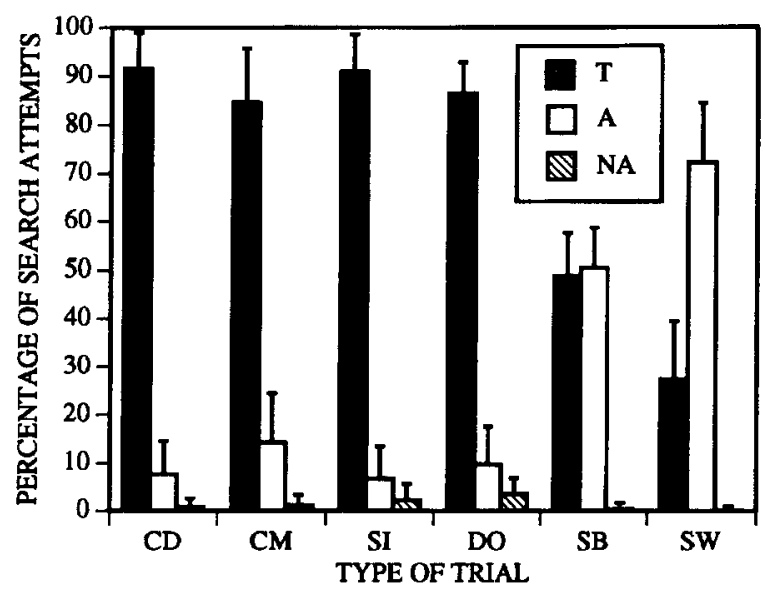

Figure 6. Mean percentage of search attempts at the target screen ( $T$ ), at the adjacent screen (A), and at the nonadjacent screen (NA) in the six types of trials of Experiment 2 with dogs. $\mathrm{CD}$, control for delay; $\mathrm{CM}$, control for movement; SI, single transposition; DO, double transposition; SB, substitution transposition; $\mathbf{S W}$, switch transposition.
When there was direct perceptual evidence that the object could no longer be at its initial position (double transpositions), working memory was reset and dogs generally chose the correct screen. When the perceptual cue indicating that the object's position had changed was only indirect (substitution transpositions), dogs tended to search for the object at the empty adjacent screen as often as they did at the target screen. The object's initial position was not cancelled and both the initial and final positions were equally activated in working memory. Finally, when there was no perceptual cue indicating that the object's position had changed (switch transpositions), dogs searched for the object more frequently at its initial than at its final location. The former location was more activated in working memory than the latter.

\section{GENERAL DISCUSSION}

The invisible transposition problems used in this research reveal several similarities between cats and dogs. First, although the screens could be easily discriminated by their dimensions and/or by the stimuli displayed on their front panels, cats and dogs did not use this information. In both species, search behavior was based on processing of spatial information rather than on recognition of the visual features of the hiding locations themselves. Second, both species reached a high level of success if and only if there was direct and strong perceptual evidence that the object could not be hidden at its initial position. Search behavior in double transpositions suggested that they find the object by looking at the screen nearest to the object's initial position and in the direction of the movement they saw during the manipulation. Third, unlike 20 -month-old infants (Sophian, 1985), cats and dogs were unable to succeed in switch transpositions and to predict the new position of the hidden object by inferring its movement.

These behavioral similarities between cats and dogs suggest that Goulet et al.'s (1994) interpretation of cats' search behavior may also apply to dogs. The disappearance of an object behind a screen activates this location in working memory, and the activation remains strong unless the object is visibly removed or, we may now add, unless the object visibly can no longer be at this location. In substitution and in switch transpositions, the object was not seen leaving its initial position, and because another screen filled this position after the manipulation, there was no direct visual cue indicating that the object could not occupy this position. Therefore, in these trials, the object's initial position was still strongly activated in working memory.

The major difference between cats and dogs appeared in substitution transpositions. Dogs were more successful in these trials than in switch transpositions, whereas cats' performance did not differ and was poor in both types of trials. Error distributions also revealed that in substitution transpositions, cats made more search attempts at the empty adjacent screen than at the target screen, and thus the object's initial position was more strongly activated in working memory than its final position. However, dogs 
made as many search attempts at the target as at the adjacent empty screen, showing that the object's initial and final positions were equally activated. It seems that dogs, unlike cats, were able to use the indirect perceptual cue provided by the change in the configuration of screens that occurred during the manipulation. Although this indirect cue did not help them in reaching a high level of success, it reduced the activation of the object's initial position in working memory and facilitated searching for the object at a different position.

The influence of an indirect perceptual cue on cats' and dogs' search behavior and on the activation in memory of the object's different locations suggests an explanation for the interspecific difference of performance between these animals in Piagetian invisible displacement tests of object permanence (Doré \& Goulet, 1992). When the empty container is shown to the subjects at the end of a trial, dogs, but not cats, can use this information, which modifies the relative activation of the two successive hiding locations (container and target screen) in working memory. Consequently, even though dogs are unable to infer the invisible movements of an object, they can reach a modest level of success in these tests, which cats fail. As a further confirmation of this interpretation, it would be interesting to compare cats' and dogs' search behavior in the new procedure for invisible displacement developed by Goulet et al. (1994).

\section{REFERENCES}

DORÉ, F. Y. (1986). Object permanence in adult cats (Felis catus). Journal of Comparative Psychology, 100, 340-347.

DoRÉ, F. Y. (1990). Search behavior of cats (Felis catus) in an invisible displacement test: Cognition and experience. Canadian Journal of Psychology, 44, 359-370.
Doré, F. Y., \& Goulet, S. (1992). Cognition chez les mammifères carnivores (chats, chiens) [Cognition in carnivorous mammals: Cats and dogs]. Psychologie Française, 37, 65-72.

Dumas, C., \& DoRÉ, F. Y. (1989). Cognitive development in kittens (Felis catus): A cross-sectional study of object permanence. Journal of Comparative Psychology, 103, 191-200.

FISET, S., \& DoRÉ, F. Y. (1990). Encodage égocentrique chez le chat domestique (Felis catus) [Egocentric spatial encoding in the domestic cat]. Paper presented at the annual meeting of the Société Québécoise pour la Recherche en Psychologie, Montréal, Canada.

FISET, S., \& DORÉ, F. Y. (1993). Spatial working memory in cats: Searching for a hidden object in a detour task. Paper presented at the annual meeting of the Canadian Society for Brain, Behaviour and Cognitive Sciences, Toronto, Canada.

GAGNON, S., \& DoRÉ, F. Y. (1992). Search behavior in various breeds of adult dogs (Canis familiaris): Object permanence and olfactory cues. Journal of Comparative Psychology, 106, 58-68.

GAGNON, S., \& DORÉ, F. Y. (1993). Search behavior of dogs (Canis familiaris) in invisible displacement problems. Animal Learning \& Behavior, 21, 246-254.

GaGNON, S., \& DoRÉ, F. Y. (1994). A cross-sectional analysis of object permanence development in dogs (Canis familiaris). Journal of Comparative Psychology, 108, 220-232.

Goulet, S., DoRE, F. Y., \& Rousseau, R. (1994). Object permanence and working memory in cats. Journal of Experimental Psychology: Animal Behavior Processes, 20, 347-365.

Howell, D. C. (1988). Statistical methods for psychology (2nd ed.). Boston: Duxbury.

Pasnak, R., Kurkian, M., \& Triana, E. (1988). Assessment of Stage 6 object permanence. Bulletin of the Psychonomic Society, 26, 368-370.

SopHIAN, C. (1985). Understanding the movements of objects: Early developments in spatial cognition. British Journal of Developmental Psychology, 3, 321-333.

Thinus-Blanc, C., Poucet, B., \& Chapuis, N. (1982). Object permanence in cats: Analysis in locomotor space. Behavioral Processes, 7 , 81-86.

Triana, E., \& PASNAK, R. (1981). Object permanence in cats and dogs. Animal Learning \& Behavior, 9, 135-139.

(Manuscript received June 24, 1994; revision accepted for publication May 26, 1995.) 